Human Ethology Bulletin 31 (2016)4: 5-14

Research Article

\title{
"PARTING IS SUCH SWEet SorRow", BUT ONLY FOR HUMANS?
}

\author{
William C. McGrew ${ }^{1} \&$ Lucy Baehren $^{2}$ \\ ${ }^{1}$ School of Psychology and Neuroscience, University of St. Andrews, UK \\ ${ }^{2}$ Department of Archaeology and Anthropology, University of Cambridge, UK
}

wcm21@cam.ac.uk

"Parting is such sweet sorrow..." (Shakespeare, 1599)

\begin{abstract}
Homo sapiens show greeting rituals when they meet and leave-taking rituals when they part. Presumably this reflects the species' fission-fusion social organisation, and such displays show notably symmetrical form and content. But what about non-humans? Here we seek in our nearest living relations (Pan troglodytes) these behavioural complexes in two ways: We report frequencies of meeting and parting in daily life and solicit data on greeting and leave-taking from field sites of long-term study of these apes. Chimpanzees greet emphatically but show no leave-taking behaviour. This lack of symmetry in our nearest living relations (as well as in other animals) suggests that human greeting and leave-taking may be unique.
\end{abstract}

Keywords: Chimpanzee, Pan troglodytes, greeting, parting, fission-fusion social system, human uniqueness 


\section{INTRODUCTION}

Human beings greet one another when they meet and take leave of (bid farewell to) one another when they part. These behaviours seem to be a human universal (Brown, 1991, 2000; Eibl-Eibesfeldt, 1975, 1989; Morris, 1977; Pinker, 2002). The behavioural elements of these personal rituals or ceremonies (called by Morris, 1977, "salutation displays") have so much in common (e.g. kiss, embrace, bow, wave, etc.) that they appear to be symmetrical 'book-ends' to face-to-face encounters. However, all of the sources cited above give more space to describing greeting than leave-taking, and there seem to be fewer focussed studies on the latter (Albert \& Kessler, 1978). There is rich nuanced variation in the particular performances of these rituals across human cultures (Eibl-Eibesfeldt, 1975, 1989; Firth 1972), and they seem to be an important part of daily life.

Such rituals may arise from our fission-fusion social system, that is, over the course of a typical day, an individual leaves and rejoins others according to what needs to be done. The patterning of this coming and going presumably reflects basic variables such as age, gender, status, role, task, etc., although we know of no analysis that specifically elucidates the (presumably) interactive effects of these variables. Even in the simplest kind of human subsistence, hunter-gatherers typically practice central-place foraging that re-unites hunters and gatherers at the end of the working day (Marlowe, 2010). This pattern suggests that leave-taking and greeting have origins that go back to our hominin ancestry and so are part of human nature.

But what about non-humans? Many vertebrates, especially birds and mammals, show reunion ceremonies, especially at the nest or den, often borrowing patterns from sexual or appeasement contexts (Eibl-Eibesfeldt, 1975). Cetaceans have especially rich and varied greeting ceremonies, e.g. orcas (Orcinus orca) groups with overlapping ranges have community-specific greetings (Whitehead \& Rendell, 2015). But the best-known and described greetings are by chimpanzees, as described by Goodall (1968); behavioural elements include: embrace, kiss, hold hands, touch, bow, bob, crouch, present, mount, and genital inspect. For males, greeting may include ritualised aggressive display with bipedal swagger, stamp, shoulder hunch, bristle, and vocalisation. Goodall recorded the gestures and postures of 1065 greetings, shown by all age-sex classes, but with differences in elements among them. Her description of greeting has been replicated in many other chimpanzee populations (e.g. Nishida et al., 2010).

But counterpart rituals in parting appear to be uncommon or perhaps non-existent in non-human species. Stewart \& Harcourt (1994) described vocalisations in mountain gorillas (Gorilla g. berengei) that apparently presaged impending departure, but these occurred during rest periods that ended with the group departing in coordinated movement, not separating. Similarly, sacred baboon (Papio hamadryas) males do a quick presentation of the buttocks to one another, before walking away. Kummer (1971, p. 24) coded this as notification of one's imminent departure, as it eventually leads to the troop following suit. For chimpanzees, de Waal (2016) described a single instance of an adult female taking leave 
of various other group members, before going indoors, leaving them outdoors, in Arnhem Zoo.

Such asymmetry would be notable if it exists, especially in species with fission-fusion social organisation, but data are absent. Aureli et al.'s (2008) comprehensive review of nonhuman primate fission-fusion devotes only a few sentences to meeting and parting in their 28-pages, plus supplementary information.

Fission-fusion occurs in various forms in non-human primates: In species such as sacred or gelada baboons (Theropithecus gelada), society is multi-levelled, that is, one-male units (harems) are embedded in clans, which are embedded in troops, which are embedded in bands/sleeping aggregations (Schreier \& Swedell, 2009). Their fissioning occurs during wide-ranging daily foraging but these subgroups fuse at the end of the day for overnight sleeping security from nocturnal predators. In species such as chimpanzee (Pan troglodytes) or spider monkeys (Ateles spp.), individuals may range alone or in subgroups (parties) of varying size, for highly variable lengths of time (Symington 1990). For example, Tutin (1979) showed that a male and female chimpanzee in a mating consortship may range together, in seclusion from others, for weeks at a time, before rejoining the rest of the community. Managing social relations in fission-fusion societies seems much more challenging than in those in which all members are constantly together, e.g. fission fusion includes the option of solitude.

Studies of fission-fusion in chimpanzees are remarkably few and thin, given this unusual but key feature of social organisation. They tend to focus on two dependent variables: party size (number of participants) and composition (by age, sex, reproductive state, social rank, etc.). Various independent variables have been hypothesised and tested as determinants of variation in parties: food supply, predation, season, demography, etc. Results are mixed: In every chimpanzee population that has yielded sufficient data, presence of one or more females in oestrus increases party size (e.g. Matsumoto-Oda, 2002). Another basic variable, fruit abundance, shows significant variation across populations (Hashimoto et al., 2003).

Virtually absent are studies of the dynamics of fission-fusion in wild chimpanzees, that is, what proximate variables cause an individual to join or to leave others, in the course of a day's activity. Or, what causes some individuals to stay together more regularly and others to avoid one another. We lack even basic descriptive data on how many fissions or fusions occur in a typical chimpanzee's day. Or how long parties last as a function of (say) their constituence. Or what proportion of fissions and fusions are done individually versus synchronously with others. Some preliminary research has been done: Blackburn and McGrew (2013) used data from Gombe to see if fission-fusion dynamics related to feeding competition; they found that chimpanzee parties fissioned for feeding, then fused afterwards. Aureli and Schaffner (2007) studied causal dynamics in wild spider monkeys, which showed conflict management (aggression versus affiliation) when subgroups fused.

Studies of wide-ranging species such as primates are difficult to do in captivity where free association or avoidance of it is limited by confinement. Greeting can be studied either experimentally (by enforced separation, e.g. Matheson et al., 1996) or observationally, but 
rarely are subjects separated in all sensory modalities. Thus, visual separation does not mean acoustic separation (Okamoto et al., 2001). Regardless of these constraints, many behavioural elements of greeting and their functional outcomes persist: Chimpanzees at Japan's Primate Research Institute kiss and embrace in affiliation at reunion, independent of the sexes involved. But just as not all fission-fusion leads to greeting and parting, not all greeting occurs in fission-fusion: Guinea baboons (Papio papio) at Chicago's Brookfield Zoo, maintained in a single group, showed greeting when one approached another, apparently as a test of social bonds (Whitham \& Maestripieri, 2003). We have found no reports of habitual leave-taking in parting in captive primates, however.

The aim of this paper is to see if humankind's nearest living relation, the chimpanzee, shows both meeting and greeting and parting and leave-taking in nature. To investigate this requires establishing that wild chimpanzees show sufficiently frequent normative fissionfusion, and if so, whether they greet when they meet and take leave when they part, on a species-wide basis. The null-hypothesis is the usual one of no difference between closelyrelated species in the same clade, until evidence shows otherwise.

\section{METHOD}

\section{Behavioural data}

We extracted data on meeting and parting by adult and adolescent chimpanzees from the standard, background data-collection instrument at Gombe, the Travel-and-Group (T\&G) chart. T\&G data range in duration from all-day (nest-to-nest, ca. 12-13 hr) to fractions of the day (for a previous analysis done similarly, see Lodwick et al. 2004). Thus, a T\&G may start in the morning at arising from the overnight nest or finish in the evening when retiring to the new nest, or both, or any fraction of the day in between those points. These focalsampling data include records of when a focal chimpanzee meets or departs from others, timed to the nearest $5 \mathrm{~min}$, thus they indicate the formation, modification and dissolution of parties, as the core of the species' fission-fusion social structure.

For each follow, we recorded its start and end, giving its duration in min. We sought to standardise the analysis while at the same time maximising the amount of data by taking the 10 longest (duration) follows of each subject. The overall average follow lasted $438 \mathrm{~min}$ $(\mathrm{SD}=111)$. Within each follow, each meeting and parting event involving the focal subject and one or more other individuals yielded frequency data. Thus, we calculated a mean rate of meeting and parting for each focal individual, and these rates were used in non-parametric statistical sampling, with level of significance (alpha) $=0.05$, two-tailed.

For this pilot study, we used archival data collected from 1972-75. We extracted data only from adults (who were independently moving, unlike dependent offspring) who had at least 10 follows (seeking to avoid small sample size effects). From 41 subjects with focalsubject follows, we used data from 23 subjects ( 13 females, 10 males), yielding a total of 230 follows. This sample is only a small proportion of the total $T \& G$ data set, which has been 
collected over decades (see Strier et al. 2010), so our study should be considered preliminary.

Please note that $T \& G$ data provide no information on greetings or leave-takings shown at meetings or partings, but reveal only the fission-fusion opportunities for showing such behaviour.

\section{Questionnaire}

We sought to establish the prevalence of meeting and greeting and of parting and leavetaking in wild chimpanzees by asking two simple questions of experienced field researchers at the 10 field sites at which these apes are followed from nest-to-nest (that is, all-day follows from dawn to dusk). These are: Bossou (Republic of Guinea), Budongo (Uganda), Fongoli (Senegal), Gombe (Tanzania), Goualougo (Republic of Congo), Kalinzu (Uganda), Kanyawara (Uganda), Mahale (Tanzania), Ngogo (Uganda), Tai (Ivory Coast). We got supplemental information from other sites but non-systematically.

We asked recipients of our enquiry to answer two simple questions: "In your chimpanzee study population, do your apes show greeting behaviour when they meet after being apart?" and "In your chimpanzee study population, do your apes show leave-taking/farewell behaviour when they part after being together?". We asked them to answer from a choice of four options: Absent (=not seen), Present (=neither habitual or customary but clearly identified), Habitual (=seen repeated in several individuals), and Customary (=seen in all or most able-bodied members of at least one age-sex class). These coding categories were taken from Whiten et al.'s (1999) study of chimpanzee cultures.

We gave recipients no definitions for any of the terms included in the questions, preferring not to prejudice or constrain their responses. We made no mention of our hypotheses but promised to brief them later. We sent requests to multiple researchers from some sites, for reliability checking and in case potential respondents might be absent doing field work (as turned out to be the case). We asked respondents to add any comments if they wished, and many did so.

\section{RESULTS}

\section{Behavioural data}

Gombe adult chimpanzees averaged 8.6 meetings (S.D. $=2.7$, range $=3.3-17.2$ ) and 6.7 partings (S.D.= 1.8, range $=2.1-10.2$ ) per day (based on Lodwick et al.'s, 2004, findings of average active day-length of $714.5 \mathrm{~min}$ ). In hourly terms, this translates to averages of 0.74 meetings and 0.52 partings per hour. Thus, chimpanzees meet and part often during the day, but they do more meeting than parting (Wilcoxon, $\mathrm{n}=23, \mathrm{z}=-3.806, \mathrm{p}=0.00$ ). This difference holds for both females and males. No differences emerged between the sexes in mean length of follow (Mann-Whitney $U$ test, $n 1=10, n 2=13, U=48, p=0.29$ ), frequency 
of meeting $(U=50, p=0.35)$ or parting $(U=41.5, p=0.15)$, or rate of meeting $(U=50, p$ $=0.35)$ or parting $(\mathrm{U}=48.5, \mathrm{p}=0.31)$.

However, while males showed no correlation between their rates of meeting vs. parting (Spearman, $\mathrm{n}=10, \mathrm{rs}=0.54, \mathrm{p}=0.11$ ), females showed a significantly higher correlation $(\mathrm{n}=13, \mathrm{rs}=0.615, \mathrm{p}=0.025)$. This sex difference may be related to female reproductive state: For females ranked by sexual swelling state (5-point scale of 0-4), the greater the swelling, the higher the rate of meeting $(n=13, r s=0.61, p=0.027)$, but there was no relation between swelling state and rate of parting $(r s=0.24, p=0.44)$. Thus, receptive females seemed to attract and retain males.

Taken together, these results show that wild chimpanzees at Gombe have plenty of opportunities for both greeting and leave-taking.

\section{Questionnaire data}

We received responses from 16 field workers from the 10 study sites (range from any one site: $1-5)$. The results were strikingly asymmetrical: At all 10 sites, the consensus was that greeting is habitual $(n=2)$ or customary $(n=8)$. All of the supplementary comments were congruent with Goodall's (1968) description of greeting behaviour. Thus, greeting appears to be a chimpanzee universal.

For leave-taking, the consensus (all 10 sites) was that it is absent. However, some respondents indicated uncertainty, apparently for two reasons: Lack of being given an operational definition meant that some were cautious about being able to exclude leavetaking altogether; and the logical problem of proving the negative, that is, of showing conclusively that a phenomenon is absent. For that reason, their caveats are presented and explored here: Only one respondent classed leave-taking as customary or habitual in their study population, saying that "... leave-taking can be signified by going half way down a tree and sitting there, others usually follow when they are ready." (See above for notification of departure.) However, two others studying the same chimpanzees rated leave-taking as absent. Similarly, only one respondent studying another population classed leave-taking as present, based on a single, putative case. Two others studying the same population classed it as absent. All others reported never having seen leave-taking. Thus, leave-taking in chimpanzees appears to be universally absent.

\section{DISCUSSION}

Why do chimpanzees greet with fervor but eschew leave-taking? More precisely, why do our nearest living relations show such binary, presence/absence asymmetry, while humans everywhere show symmetric sandwiching of their encounters? Perhaps Shakespeare (1599) provided a clue, in the complete quotation from Juliet: "Parting is such sweet sorrow that I shall say good night till it be morrow." (Act 2, Scene 2, italics ours). Perhaps the pertinent issue is anticipation, that is, 'mental time-travel' (chronesthesia) forward (Cheke \& Clayton 2010). Such cognition is well-known in some non-humans, for example, in the caching 
behaviour of corvids and rodents (e.g. Raby et al. 2007). Similarly, chimpanzees show anticipatory behaviour, but all of the examples we know of in these apes concern physical objects: An adult male in Copenhagen Zoo spontaneously hid projectiles from keepers, so that he could throw them the next day at zoo visitors (Osvath and Karvonen 2012). Orangutans and bonobos in controlled experiments saved appropriate tools overnight for use the next day (Mulcahy \& Call 2006). However, we know of no studies of social anticipation in apes, nor of any easy way that it could be directly studied.

Perhaps the difference between meeting and parting lies in the costs and benefits of the selective forces inherent in chimpanzee fission-fusion. Being seperated from others entails mutual ignorance of what others have been doing in one's absence; for a hierarchical species, it could mean, for example, important changes in social rank relations. The longer the separation, the greater the possibility of key events having been missed (which yields a simple hypothesis that the longer the interval between last parting and first meeting, the more important and therefore more pronounced will be any greeting, all other things being equal). The very different metrics of minutes, hours, days, or weeks of absence could present crucially different social challenges in meeting. All in all, good reasons exist for meeting and greeting to be socially charged.

But what about parting? In species that fragment daily, such as hamadryas baboons, only to reunite at the end of the day for overnight, aggregational sleeping, the period of separation is never more than hours, and reunion is highly predictable (Kummer 1971). Thus, separation after parting in the morning when the group disperses from the sleeping site means little, as daily foraging lasts no more than about 12 hours. But when two chimpanzees part, neither likely knows how long it will be before they meet again; it seems likely that no crucial information is exchanged. Perhaps this is just too great a cognitive challenge, trying to predict when the next meeting is likely to occur, based on multiple variables ranging as widely as reproductive cyclicity and resource ephemerality. Or, perhaps, at a deeper cognitive level, it suggests a lack of self-knowledge of mortality, allowing chimpanzees to live their lives without foreboding (Anderson 2016). Parting per se seems mundane and uninformative, compared to meeting.

From these results, meeting and greeting should be more frequent and conspicuous than parting and leave-taking, all other things being equal. The former has obvious informative function, while the latter seems to be of minor importance. Of course, one can speculate about the possible utility of leave-taking: Chimpanzee males form alliances that affect their competition with others, e.g. social dominance acquisition and retention. Knowledge of presence or absence of a key ally may be crucial in social success or failure. These possibilities require empirical testing.

When evolutionary psychologists and human ethologists seek qualitative differences between humans and non-humans, including our nearest living relations, they often focus on the obviously big behavioural phenomena, such as language, culture or bipedal locomotion. Often, after scrutiny, these turn out to be only quantitative differences. Here, we have explored such potential human uniqueness at a much more modest and proximal 
level, in the regular comings and goings of daily life. Further empirical studies are needed to see if our tentative findings of a difference in kind (rather than just a difference of degree) hold up. Especially useful would be ethological studies of spontaneous leave-taking in human everyday life, which might help to distinguish mere social conventions from useful, even essential, behaviour.

\section{ACKNOWLEDGEMENTS}

We thank: Fieldworkers for providing questionnaire data: Susana Carvalho, Chie Hashimoto, Catherine Hobaiter, Kimberley Hockings, Michael Huffman, Lydia Luncz, Tetsuro Matsuzawa, John Mitani, David Morgan, Naruki Morimura, Robert O’Malley, Jill Pruetz, Vernon Reynolds, Janette Wallis, Richard Wrangham; Caroline Tutin for access to Gombe data from her COPS study; James Anderson and Adam Kendon for helpful input.

\section{REFERENCES}

Albert, S. \& Kessler, S. (1978) Ending social encounters. Journal of Experimental Social Psychology, 14, 541-553. DOI

Anderson, J.R. (2016) Comparative thanatology. Current Biology, 26, R543-576. DOI

Aureli, F. \& Schaffner, C.M. (2007) Aggression and conflict management at fusion in spider monkeys. Biology Letters, 3, 147-149. DOI

Aureli, F., Schaffner, C.M., Boesch, C., Bearder, S.K., Call, J., Chapman, C.A., et al. (2008) Fissionfusion dynamics. New research frameworks. Current Anthropology, 49, 627-654. DOI

Blackburn, A. \& McGrew, W.C. (2013) Fission-fusion in chimpanzees: feeding as a proximal mechanism at Gombe. Pan Africa News, 20, 19-22.

Brown, D.E. (1991) Human universals. New York: McGraw-Hill.

Brown, D.E. (2000) Human universals and their implications. In N. Roughley (ed.), Being human: Anthropological universality and particularity in transdisciplinary perspectives. (pp 156-174) New York: Walter de Gruyter. DOI

Cheke, L.G. \& Clayton, N.S. (2010) Mental time travel in animals. WIREs Cognitive Science, 1, 915930. DOI

Eibl-Eibesfeldt, I. (1975) Ethology. The biology of behavior. New York: Holt, Rinehart \& Winston.

Eibl-Eibesfeldt, I. (1989) Human ethology. New York: Aldine de Gruyter.

Firth, R. (1972) Verbal and bodily rituals of greeting and parting. In J.S. La Fontaine (ed.), The interpretation of ritual. (pp 1-38) London: Tavistock. 
Goodall, J.v.L. (1968) The behaviour of free-living chimpanzees in the Gombe Stream Reserve. Animal Behaviour Monographs, 1, 161-301. DOI

Hashimoto, C., Suzuki, S., Takenoshita, Y., Yamagiwa, J., Basabose, A.K., \& Furuichi, T. (2003) How fruit abundance affects the chimpanzee party size: a comparison between four study sites. Primates, 44, 77-81.

Kummer, H. (1971) Primate societies. Group techniques of ecological adaptation. Chicago: Aldine.

Lodwick, J.L., Borries, C., Pusey, A.E., Goodall, J., McGrew, W.C. (2004) From nest to nestinfluence of ecology and reproduction on the active period of adult Gombe chimpanzees. American Journal of Primatology, 64, 249-260. DOI

Marlowe, F.W. (2010) The Hadza Hunter-Gatherers of Tanzania. Berkley: University of California Press.

Matheson, M.D., Johnson, J.S. \& Feuerstein, J. (1996) Male reunion displays in tufted capuchin monkeys (Cebus apella). American Journal of Primatology, 40, 183-188. DOI

Matsumoto-Oda, A. (2002) Social relationships between cycling females and adult males in Mahale chimpanzees. In C. Boesch, G. Hohmann, L.F. Marchant (Eds.), Behavioural diversity in chimpanzees and bonobos (pp 168-180). Cambridge: Cambridge University Press. DOI

Morris, D. (1977) Manwatching. A field guide to human behaviour. St. Albans: Triad/Panther.

Mulcahy, N.J. \& Call, J. (2006) Apes save tools for future use. Science, 312, 1038-1040. DOI

Nishida, T., Zamma, A., Matsusaka, T., Inaba, A., \& McGrew, W.C. (2010) Chimpanzee behaviour in the wild. An audio-visual encyclopedia. Tokyo: Springer.

Okamoto, K., Agetsuma, N., \& Kojima, S. (2001) Greeting behaviour during party encounters in captive chimpanzees. Primates, 42, 161-165. DOI

Osvath, M. \& Karvonen, E. (2012) Spontaneous innovation for future deception in a male chimpanzee. PLoS One, 7, e36782. DOI

Pinker, S. (2002) The blank slate. The modern denial of human nature. New York: Penguin.

Raby, C.R., Alexis, D.M., Dickinson, A., Clayton, N.S. (2007) Planning for the future by western scrub-jays. Nature, 445, 919-921. DOI

Schreier, A.L. \& Swedell, L. (2009) The fourth level of social structure in a multi-level society: ecological and social functions of clans in hamadryas baboons. American Journal of Primatology, 71, 948-955. DOI

Shakespeare, W. (1599) Romeo and Juliet. London: Second Folio.

Stewart, K.J. \& Harcourt, A.H. (1994) Gorillas' vocalizations during rest periods: signals of impending departure? Behaviour, 130, 29-40. DOI

Strier, K.B., Altmann, J., Brockman, D.K., Bronikowski, A.M., Cords, M., Fedigan, L.M. et al. (2010) The Primate Life History Database: a unique shared ecological data resource. Methods in Ecology and Evolution 1:199-211. DOI 
Symington, M.M. (1990) Fission-fusion social organization in Ateles and Pan. International Journal of Primatology, 11, 47-61. DOI doi: 10.1007/BF02193695

Tutin, C.E.G. (1979) Mating patterns and reproductive strategies in a community of wild chimpanzees (Pan troglodytes schweinfurthii). Behavioral Ecology and Sociobiology, 6, 29-38. $\underline{\text { DOI }}$

de Waal, F.B.M. (2016) Are we smart enough to know how smart animals are? New York: W.W. Norton.

Whitham, J.C. \& Maestripieri, D. (2003) Primate rituals: the function of greetings between male Guinea baboons. Ethology, 109, 847-859. DOI

Whitehead, H. \& Rendell, L. (2015) The cultural lives of whales and dolphins. Chicago: University of Chicago Press. DOI 